\title{
A study on conceptual transfer in the use of prepositions in English writing by Chinese secondary school students
}

\begin{abstract}
This study addresses the issue of conceptual transfer in Chinese EFL learners' use of prepositions under the guidance of Image Schema Theory, aiming to explore the cognitive underpinnings of conceptual transfer. By observing linguistic data from the learner corpus WCEL (Writing Corpus of English Learners), part of ICCI (The International Corpus of Crosslinguistic Interlanguage), this study summarises types and manifestations of conceptual transfer in the use of prepositions in English writing by Chinese secondary school students, and analyses corresponding cognitive causes of conceptual transfer. Data processing software, AntConc, is used for observation of concordance lines according to the minimum assumption proposed by Sinclair (2004) in corpus-based studies. It is found that errors made by students in their use of English prepositions are mainly caused by negative conceptual transfer of the Chinese language; positive conceptual transfer also exists. Conceptual transfer is mainly caused by cognitive similarities and differences between English and Chinese, represented by image schemas.
\end{abstract}

Key words: conceptual transfer, image schema, prepositions, English writing, Chinese secondary school students.

\section{Introduction}

Language transfer has been a central issue in second language acquisition (SLA) research. It is not only the result of language learning, but also a process in which human cognition and conceptualisation are involved (Odlin 1989). Conceptual transfer (Jarvis and Pavlenko 2008) emphasises the role of human cognition and conceptualisation in the process of language acquisition. Learners' writing can be regarded as linguistic representations of their linguistic competence, and therefore serves as primary and valid data for studies in the field of SLA. Among the linguistic units that L2 learners need to acquire, prepositions used in the writings of L2 learners are related to temporal and spatial concepts. The use of prepositions reveals cognitive processes and can be analysed

\footnotetext{
1 First author

2 Corresponding author
} 
to investigate conceptual transfer. With these considerations in mind, this study aims to explore types and manifestations of conceptual transfer in the use of prepositions in English writing by Chinese secondary school students, and tries to explain the conceptual transfer with an emphasis on the conceptual similarities and differences between Chinese and English.

\section{Literature Review}

\subsection{Language transfer and conceptual transfer}

The term "language transfer" can be used interchangeably with "cross-linguistic influence", denoting the existence of influence across different languages. It is not easy to find an all-inclusive definition of language transfer. Among existing definitions of language transfer, the working definition by Odlin (1989) has shown more about the nature of language transfer. According to Odlin, language transfer refers to the influence resulting from the similarities and differences between the target language and any other language that has been previously (and perhaps imperfectly) acquired.

Language transfer can be classified into linguistic transfer, semantic transfer and conceptual transfer, corresponding to the transfer of linguistic elements (e.g. pronunciation and syntactic structure), meanings and concepts (Zhang and Liu 2013). Currently, little attention has been paid to conceptual transfer (Jarvis and Pavlenko 2008). Conceptual transfer can be understood at three different levels: as an observation, approach and hypothesis since it "focuses more on the effects of cognition on language use, particularly the effects of patterns of cognition acquired through one language on the receptive or productive use of another language" (Jarvis 2011: 3). In SLA, conceptual transfer refers to the influence of one language on the acquisition of another in terms of thinking patterns, in which process the way L2 learners think through L1 and their mode of thinking through L2 interact with each other (Jarvis and Pavlenko 2008).

\subsection{Relevant studies of language transfer and conceptual transfer}

Investigations of language transfer mainly include phonetic, phonological, lexical, morphological, syntactic, semantic, pragmatic as well as conceptual studies, which reveal different dimensions and provide convincing evidence of language transfer. Existing studies of transfer may overlap and thus can be categorised roughly into three major types, i.e. linguistic transfer, semantic transfer and conceptual transfer. Most early studies are concerned with linguistic transfer, which may be phonetic, phonological, lexical, morphological, or syntactic. Some studies (e.g. Hancin-Bhatt 2000, Aoyama et al. 2004) deal with the acquisition of L2 phones and phonemes. Some (e.g. Ringbom 2001) discuss the use of L2 vocabulary and likewise show cross-linguistic influence. There are also studies (e.g. Jarvis and Odlin 2000, Koda 2000) dealing with morphological transfer. Certain syntactic structures have also been examined in the field (e.g. Matthews and Yip 2003). Both semantic transfer and pragmatic transfer deal with cross-linguistic influence in meaning, which may overlap with but do not equal conceptual transfer (e.g. Ijaz 1986, Kwon 2003, Tamanaha 2003). 
Conceptual transfer can be regarded as a subset of semantic transfer in accordance with the claim that conceptual transfer involves semantic transfer, not vice-versa (Odlin 2005). There are discussions concerning the notion of conceptual transfer (e.g. Pavlenko 1999, 2002, von Stutterheim 2003) as well as empirical studies under the guidance of different theoretical frameworks. Some scholars investigated different linguistic levels of conceptual transfer within the paradigm of Cognitive Linguistics or linguistic relativity (e.g. Ijaz 1986). In accordance with Odlin (2005), conceptual transfer is concerned with a second language and can be understood as cases of linguistic relativity. Accordingly, some studies of conceptual transfer are often related to issues of relativity, which can be understood briefly as the hypothesised effect of language on thought (e.g. Pederson et al. 1998, Jarvis 1998, Pavlenko 1999). Recently, scholars in China have explored language transfer from the perspective of psychology or cognitive linguistics (e.g. Ma 2010, Xu et al. 2014). Under the influence of the Linguistic Relativity Principle, Zhang and Liu (2013b) explored features of Chinese EFL beginners' acquisition of English metaphorical prepositions by utilizing linguistic data from ICCI through the analysis of their collocations.

From the above, we can see that conceptual transfer has gained increasing attention, and it has developed rapidly, as reflected in the diverse topics. It includes not only L1 influence on L2 learning, but also bidirectional transfer. Trilingual and multilingual situations in conceptual transfer studies have been considered in some particular studies. However, there are few investigations of conceptual transfer with a consideration of Chinese EFL learners' linguistic output. In other words, very few studies have been conducted on the Chinese secondary learners. Therefore, it is necessary to conduct such a study of conceptual transfer through the analysis of Chinese EFL learners' linguistic performance.

\subsection{Image Schema Theory}

In the analysis of causes of conceptual transfer, Image Schema Theory was employed in order to compare the conceptual similarities at the lexical level between Chinese and English as far as spatial or temporal prepositions were concerned.

The concept of "image schema" is generally considered to have first been proposed in Conceptual Metaphor Theory by Lakoff and Johnson (1980). According to this theory, metaphor is fundamentally conceptual, not linguistic in nature. Although the overwhelming majority of evidence for conceptual metaphor is linguistic, it is also true that people structure their concepts metaphorical when they are not using language (Casasanto 2009). Johnson (1987: xiv) defines an image schema as "a recurring, dynamic pattern of our perceptual interactions and motor programs that gives coherence and structure to our experience", emphasizing the "perceptual experience" nature of an image schema. Gibbs and Colston (1995: 349) define image schemata as "dynamic analog representations of spatial relations and movements in space", which draws attention to the "spatial" aspect of an image schema. According to Oakley (2007), an image schema can be defined as a condensed redescription of perceptual experience for the purpose of mapping spatial structure onto conceptual structure, which is a combination of the above two, emphasizing "perceptual 
experience", "spatial structure" and "conceptual structure". These three diachronically arranged definitions vary in terms of the linguistic expressions that are employed to define the term "image schema", but the nature of this term remains the same. An image schema reveals people's concepts acquired in their interactions with the outside world. An image schema can be expressed or understood with the help of simple diagrams in the results and discussion sections. The notions "trajector (TR)" and "landmark (LM)" are essential to understanding an image schema. The former is the primary focus of attention, and the latter equals the background in a spatial relationship.

Linguistic representations in the use of prepositions reveal similarities as well as differences in terms of conceptual structures between Chinese and English. Therefore, theoretical support from Image Schema Theory cannot be neglected for its role in explaining the underlying commonalities and discrepancies between concepts related to the use of English prepositions by Chinese secondary school students in their writing, especially to the use of spatial and temporal prepositions as these prepositions reveal cognitive processes in which people interact with their physical world, social world and even psychological world.

\section{Research Design}

A corpus-based approach to learner language is perceived as an important research methodology due to authentic linguistic data and the convenient process of data processing. It is applicable to an investigation of systematic and regular features in Chinese learners' use of English prepositions by retrieving and observing their use of the target language.

\subsection{Research questions}

To investigate conceptual transfer in the use of prepositions in English writing by Chinese secondary school students at the lexical level, we focus on the following two research questions:

RQ1: What are the types and manifestations of conceptual transfer in the use of prepositions in English writing by Chinese secondary school students?

RQ2: What are the underlying causes of conceptual transfer in the use of prepositions in English writing by Chinese secondary school students?

By focusing on these two research questions, this study may provide some evidence of Chinesespecific conceptual transfer in the process of English learning. The linguistic data were chosen from WCEL (Writing Corpus of English Learners), which contains altogether 166,301 tokens of 1, 494 written documents based on essay writing. Writing samples in WCEL were collected from first- and second-year secondary school students from twelve key and ordinary secondary schools in three cities (Shenyang, Changchun and Harbin) of northeastern China. It is worth noticing that key secondary schools refer to top schools that have a better guarantee of teaching resources and excellent student enrolment compared with ordinary secondary schools. To ensure typicality of the data, two key secondary schools and two ordinary secondary schools were considered 
in each city. The students' gender was taken into consideration to balance the ratio of male and female students. They are all native speakers of Mandarin Chinese and they learn English as a foreign language in school settings. Their age ranges from 12-17 years old, and English learning experience from 4-6 years. They were required to complete their English writing tasks within 20 minutes without any assistance from teachers or dictionaries. The writing tasks used were mainly descriptive, including topics such as A Christmas party, A funny thing that happened to me, Computer games, How to make children get enough exercise, How I could spend 100 yuan, My birthday, My school, Exercise, Smoking, Watching TV. The corpus can be used to investigate the systematic features or regularities revealed in Chinese secondary school students' written English.

\subsection{Data collection and processing}

In this study the learner corpus, WCEL, was used to help identify those aspects of the students' performance in the use of English prepositions due to the influence of L1 concepts. To collect the linguistic data of students' outcome in using English prepositions, we conducted a simple retrieval of these prepositions in the corpus. Thus, concordance lines that contain these English prepositions were built, which can provide data on the properties of the students' interlanguage. The processing of linguistic data involved a process of editing concordance lines, deletion of unnecessary concordance lines if they are irrelevant to the topic concerned, creation of a plain text based on concordance lines containing prepositions, identification of conceptual transfer in the use of these prepositions, classification of the types of conceptual transfer, and explanations of conceptual transfer at the lexical level.

The processing tool of the corpus, AntConc, was used to retrieve the prepositions and observe the concordance hits containing these prepositions. Conceptual transfer in the use of prepositions can be identified by observing their own textual environment and by examining the misuses of these prepositions. The format of KWIC has been widely used in data-processing. Minimum assumption is a very important claim with methodological advantages by Sinclair (2004) in corpusbased studies. According to this assumption, only a minimum of assumptions can be held in the initial stages of study. It follows that researchers should observe textual evidence with an open mind through a process of extraction of data, and observation of data, which may be a circulatory procedure until all the linguistic data have been observed and an appropriate conclusion has been reached. Minimum assumption is a typical example of a deductive method in the context of corpus-based study, which also reflects the respect for the facts hidden in the texts to be examined. The interpretation of conceptual transfer came after the classification and identification of conceptual transfer in the use of prepositions from the perspective of Image Schema Theory.

\section{Results and Discussion}

This part presents the results and discussion of two types of conceptual transfer in the use of prepositions in English writing by Chinese secondary school students under the guidance of Image Schema Theory. The study focuses on the prepositions "at", "in" and "on", not only because of 
the high frequency of concordance hits retrieved, but also because of the evidence of conceptual transfer revealed.

\subsection{Two types of conceptual transfer in the use of prepositions}

By using the data processing software, AntConc, the frequency of use of the prepositions "at", "in" and "on" in English writing by secondary school students can be obtained. This study just focuses on conceptual transfer at the lexical level under the guidance of Image Schema Theory. Linguistic data that are evident enough to reveal conceptual transfer at the lexical level were categorised into two major types of conceptual transfer, i.e. positive conceptual transfer and negative conceptual transfer. Through observation and identification of the concordance hits containing these prepositions, cases of both positive and negative conceptual transfer were identified based on the observation of concordance lines according to the minimum assumption proposed by Sinclair (2004) in corpus-based studies.

\subsubsection{Positive conceptual transfer at the lexical level}

Positive conceptual transfer in the use of prepositions at the lexical level was found in the use of the English prepositions "at" and "in". In the use of the English preposition "at", due to positive conceptual transfer at the lexical level, no errors were made in such cases in which the English preposition "at" collocates with other verbs and adjectives, i.e. phrases such as "shout at", "look at", "smile at" and "be mad at" in the following cases:

(1) It was his tree and he shouted at him.

(2) Tim's math teacher looked at his homework and saw that he had got all his sums right.

(3) I smiled at them.

(4) I thought the teacher would be mad at me.

Positive conceptual transfer in the use of the English preposition "in" was also evident as no errors were made by learners. The following underlined instances of the use of "in" provide linguistic representations of this type of conceptual transfer:

(5) In the dream, I wore a birthday hat, smiling to my father and mother.

(6) In our school, there are many trees on the playground.

(7) In many cities, smoking is forbidden in public places.

\subsubsection{Negative conceptual transfer at the lexical level}

Negative conceptual transfer in the use of prepositions at the lexical level was found in the use of the prepositions "at" and "on". Negative conceptual transfer in the use of the English preposition "at" by Chinese secondary school students was reflected in the misuses of the English preposition "at" instead of other spatial or temporal prepositions, as illustrated in the following examples: 

(8) I often watch TV at past.
(9) My birthday is at July 11th.
(10) My school also has long history. It was set up at 1950.
(11) At afternoon, my parents and I went to western restaurant to enjoy our lunch.
(12) I studyed at middle school.
(13) One day, we were studying at the classroom.
(14) I can play games and run at the playground.

Examples (8) to (11) are related to temporal concepts, while examples (12) to (14) are concerned with spatial concepts.

Likewise, negative conceptual transfer in the use of the English preposition "on", whose semantic meaning corresponds to the Chinese “上”, was also evident when expressing spatial concepts. Observe the following instances:

(15) There are many clouds on the sky.

(16) You can see many birds flying on the sky.

(17) We can see birds on the trees.

(18) Then I sat on the chair and cried.

(19) We lay on the sofa.

(20) On P. E. class, we can play sports and relax ourselves on the playground.

(21) Finally I fell asleep on the math class.

Different from instances (15)-(19), which express abstract spatial relations, (20) and (21) are concerned with abstract spatial concepts.

\subsection{Causes of conceptual transfer in the use of prepositions}

Cognitive causes of conceptual transfer at the lexical level are analysed under the guidance of Image Schema Theory by considering conceptual similarities and differences between Chinese and English. Note that the Chinese equivalents of the prepositions discussed are provided in the Chinese original, with Pinyin and their literal English translations provided in parentheses.

\subsubsection{Cognitive causes of positive conceptual transfer at the lexical level}

Most monosyllabic characters in the Chinese language are ideographic. Chinese characters that express spatial and temporal relations indicate their meanings and conceptual categories by their original patterns or formations. This is particularly evident when it comes to Chinese characters in ancient times, e.g., inscriptions on bones or tortoise shells of the Shang Dynasty. In the process of understanding a specific spatial or temporal concept, native speakers of the Chinese language are usually influenced by the spatial or temporal concepts represented by a corresponding Chinese 
character. Consequently, in the process of learning English, transfer of L1 spatial or temporal concepts exists, and Chinese EFL learners' use of prepositions is influenced by L1 spatial or temporal concepts subconsciously.

The basic spatial conceptual meaning of the English preposition "at" is "a certain point in space", as shown in Figure 1, which is the prototypical image schema, denoting a static spatial relationship between the TR represented by an orbicular shading and the LM represented by a parallelogram.

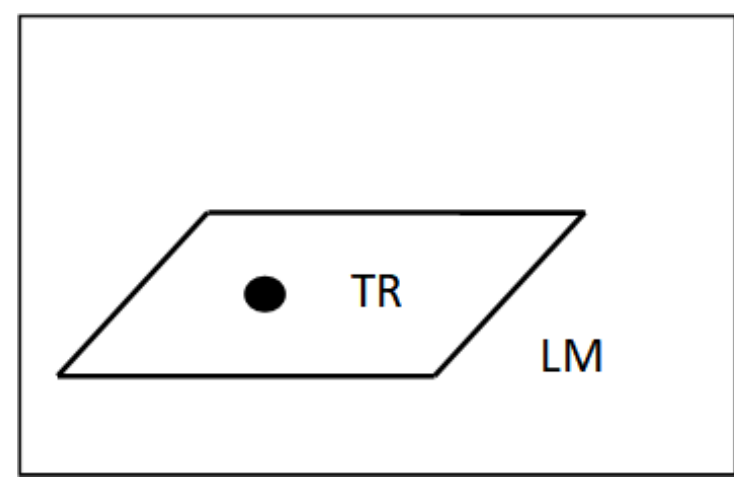

Figure 1 Static schema for "at"

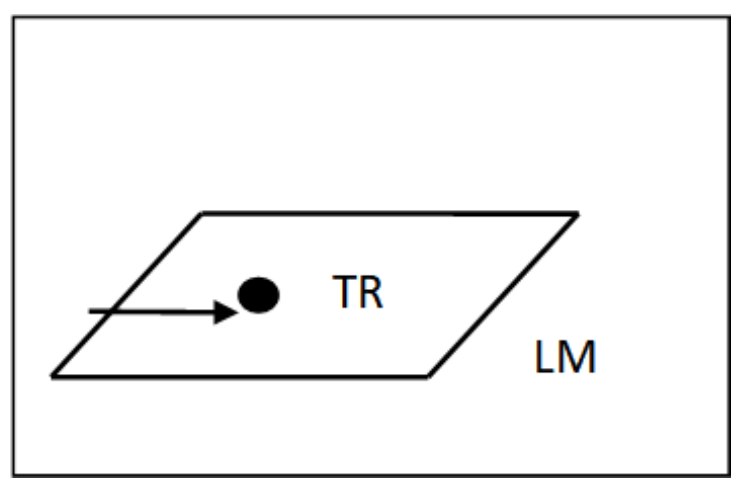

Figure 2 Dynamic schema for "at"

The semantic meaning of the English preposition "at" corresponds to the Chinese expressions “朝(chao, towards)”, “向(xiang, towards)”, “对(dui, to)”, and “面对(miandui, face)”, all of which share the meaning "facing or towards a certain direction", and is represented by a dynamic image schema signalled by an arrow $(\rightarrow)$ in Figure 2 above. In the use of the English preposition "at", positive conceptual transfer of its corresponding Chinese expressions makes secondary school students acquire the spatial meaning of "at" with ease. The spatial relation expressed by "at" in the English language is quite similar to that indicated in the corresponding Chinese expressions when it cooccurs with verbs like "shout", "look", "smile" and adjectives like "mad" to form collocations, all of which have the potential meaning of "pointing to a certain direction" and may function together with an object in the expressions within which they co-occur. Therefore, the correspondence of conceptual meanings between the English preposition "at" and the Chinese “朝(chao, towards)", “向(xiang, towards)”, ”对(dui, to)”, and “面对(miandui, face)” may lead to positive conceptual transfer. However, we have to admit that there may be other explanations, e.g., the students have used the English preposition "at" correctly perhaps because they have properly memorized them.

Conceptually, the English preposition “in” corresponds to Chinese “里(li, in)”, whose original meaning equals “裏(guo, in)” or “衣内 (yinei, the inner side of clothing)” (Xu 2001). The spatial concept represented by the Chinese character “里(li, in)" is very similar to that represented by the English preposition "in". Spatial concepts can be used metaphorically as temporal or psychological concepts, and this is true for the spatial concept “里(li, in)”. Its spatial concept can be mapped onto temporal and psychological concepts. And this mapping process is quite similar to that of the English preposition "in”. To wit, in the use of Chinese “里(li, in)" and the English preposition "in", the grammatical metaphor in the Chinese language resembles that in the English language in 
terms of conceptual mapping processes. To illustrate the conceptual attributes of “里(li, in)” and "in" more clearly, these two concepts are represented by two similar image schemas with subtle differences, as shown Figure 3 and Figure 4.

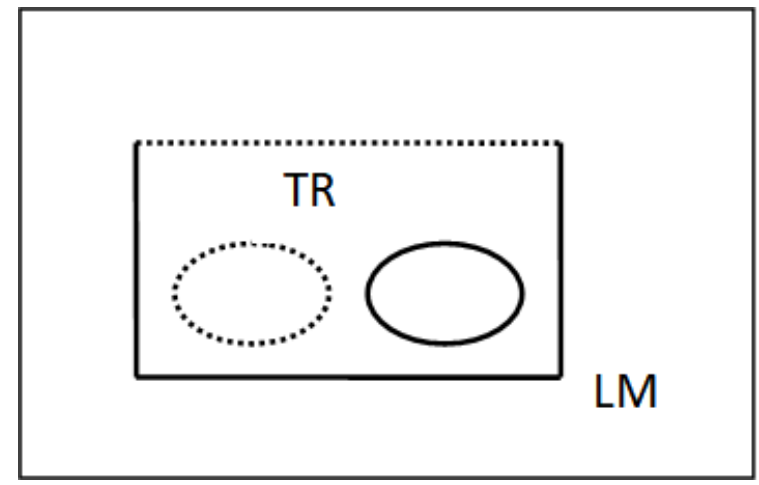

Figure 3 Image schema for “里(li in)”

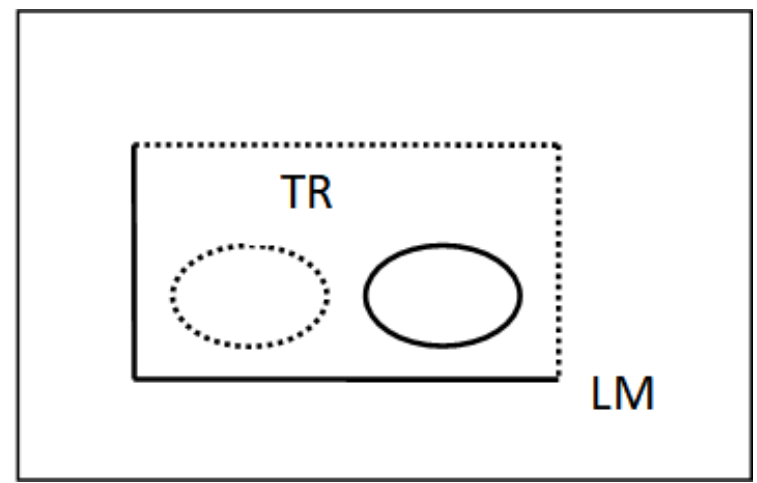

Figure 4 Image schema for "in"

The above two diagrams illustrate that the concept “里(li, in)" and the English "in" share both conceptual similarities and differences. The Chinese “里(li, in)" represents the conceptual attributes that "the TR is wholly inside the LM, which can be closed or open", and that "the TR can be moving or static". The English preposition "in" represents the conceptual attributes that "the TR is surrounded or partially surrounded by other items", and that "the TR can be moving or static". By comparison, it is clear that the only difference between these two image schemas lies in whether the TR is wholly surrounded. Therefore, the spatial, temporal, or psychological concept represented by the English preposition "in" is broader than the concept represented by the Chinese "里(li, in)". For example, in cases where students use the expressions "in the dream", "in our school" and “in many cities", the Chinese concept “里(li, in)", which is limited in its conceptual meaning compared with the English preposition "in", plays a positive role. That means, in any situations where the Chinese concept “里(li, in)" is retrieved, the use of the English preposition "in" is correct with no doubt. And this leads to positive conceptual transfer in the use of the English preposition "in" by Chinese EFL learners.

\subsubsection{Cognitive causes of negative conceptual transfer at the lexical level}

A major difference between the preposition “at" and its Chinese equivalent “在(zai, at/in/on/exist/ be)" lies in that the latter has a much wider application range due to its relatively rich conceptual attributes, which may cause negative conceptual transfer of the Chinese “在(zai, at/in/on/exist/be)" in the use of the English preposition "at".

While expressing temporal concepts, the English preposition "at" relates to an exact time. However, its equivalent Chinese “在(zai, at/in/on/exist/be)" does not have this limitation in expressing temporal concepts. And this is also true in expressing spatial concepts, in which case the English preposition "at" mainly represents "exist at a specific point, a relatively small place, or 
a place that can be regarded as a point”. However, the Chinese character “在(zai, at/in/on/exist/ be)" does not have this limitation in expressing spatial concepts. It has a much wider application range and therefore can be applied to refer to almost anything that exists in our physical or mental space. Accordingly, when Chinese EFL learners express temporal or spatial concepts, they may enlarge the application range of the English preposition "at" due to the negative influence of the Chinese concept “在(zai, at/in/on/exist/be)”. Therefore, Chinese EFL learners tend to make errors in their use of the English preposition "at" due to the negative influence of the Chinese concept “在(zai, at/in/on/exist/be)”.

In expressing concrete spatial relations, the similarities and differences between concepts lexicalised by the English preposition "on" and its corresponding Chinese character “上(shang, on/ upon/upper)" can be well understood in terms of the image schemas in Figure 5 and Figure 6, respectively.

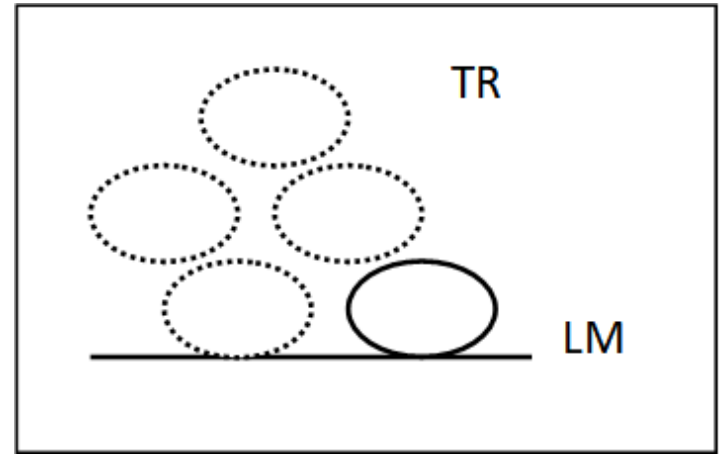

Figure 5 Image schema for “上 (shang, on/upon/upper)”

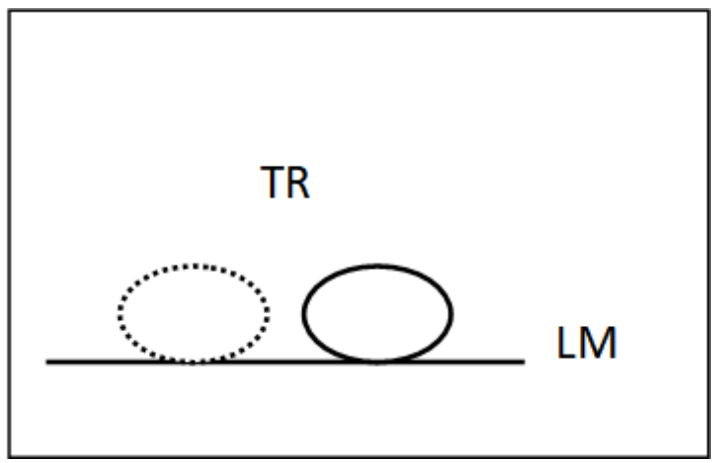

Figure 6 Image schema for "on"

The lexical concepts specifically encoded and externalised by the Chinese character “上(shang, on/upon/upper)" include that "the TR is on or above the LM", "the TR may be in a state of motion or static", and "the TR may be exposed in the air or surrounded by other items". In contrast, the spatial concepts represented by the English preposition "on" include that "the TR is on the LM (the TR touches the LM)", "the TR may be in a state of motion or static", and "the TR should be exposed in the air but not surrounded by other items". Thus, the major difference between the spatial concepts expressed by “上(shang, on/upon/upper)" and the conceptual attributes of "on" is that when using "on", the TR should be exposed in the air, and there is physical contact between the TR and the LM, while the Chinese character “上(shang, on/upon/upper)” does not possess these two conceptual attributes. In other words, the conceptual category of “上(shang, on/upon/upper)” is broader than that of "on", which is the main cause of errors made by Chinese EFL learners due to the influence of negative conceptual transfer in the use of the English preposition "on".

To make a detailed analysis, the Chinese expression “在天上(zai tian shang, in the sky)" is acceptable in cases where the sphere of human activities is regarded by native Chinese speakers as the LM, and anything above ground level is thought of as the TR. Therefore, the Chinese 
“上(shang, on/upon/upper)" is chosen to express this kind of spatial relation between the LM and the TR. The Chinese concept of "上(shang, on/upon/upper)" is close to the English preposition "on", which requires physical contact between the TR and the LM. Therefore, the expression "on the sky" in the above instances is unacceptable for native speakers of English, and the correct form should be "in the sky", which relates to a container schema.

Another example, the Chinese expression “在树上(zai shu shang, in the tree)” illustrates that native speakers of Chinese regard their sphere of activities as the LM, and anything above ground level is regarded as the TR. Errors occur due to the difference between the spatial concept of “上(shang, on/upon/upper)" and the conceptual attribute of "on". That is to say, "the TR should be exposed in the air" in the case of "on", while the Chinese character "上(shang, on/upon/upper)" does not have this spatially conceptual limitation. In this sense, anything above ground level and existing in any part of a tree can be described by “在树上(zai shu shang, in the tree)" in the Chinese language. However, the conceptual attribute of the English preposition "on" does not include the conceptual meaning that "the TR is surrounded or partially surrounded by other items".

Additionally, negative conceptual transfer can also be found in the expression of abstract spatial relations. Following in a similar vein, the difference between concepts lexicalised by the English preposition “on” and its corresponding Chinese character “上(shang, on/upon/upper)” also exits in expressing abstract spatial concepts, which may cause negative conceptual transfer. In the Chinese language, the collocation of abstract nouns and “上(shang, on/upon/upper)” functions to express the conceptual attribute that "something is in progress". However, the collocation of abstract nouns and "on" does not have this abstract spatial meaning. The English preposition "in" can be used to express this kind of abstract spatial meaning, and therefore "in" should be used in the above instances (20) and (21) instead of "on". Therefore, it is concluded that the transfer of the Chinese lexical concepts in collocation of abstract nouns and the Chinese character "上(shang, on/upon/upper)" to the use of English prepositions by Chinese EFL learners is the main cause of the errors in the above linguistic representations at the lexical level.

In both cases of “在(zai, at/in/on/exist/be)" and “上(shang, on/upon/upper)”, there is typological linguistic difference involved. What lies at the root of the cause is that the Chinese language encodes these two ideas analytically, whereas the English language encodes them synthetically (Gennari et al. 2002). Different languages may simply instantiate underlying conceptual universals in different ways and linguistic differences have cognitive consequences in some or all circumstances.

\section{Conclusion}

Both positive conceptual transfer of L1 and negative conceptual transfer of L1 can be found at the lexical level. Specifically, positive conceptual transfer in the use of the prepositions "at" and "in", and negative conceptual transfer in the use of the prepositions "at" and "on" were presented to provide evidence for the existence of conceptual transfer in Chinese secondary school students' English learning process. 
Additionally, underlying cognitive causes of conceptual transfer were identified by comparing conceptual similarities and differences between Chinese and English under the guidance of Image Schema Theory. It can be generally concluded that similarities and differences between the conceptual attributes of English prepositions and their Chinese equivalents may lead to positive conceptual transfer and negative conceptual transfer, respectively. Where there are similarities between conceptual meanings of English prepositions and their corresponding Chinese expressions, there is positive conceptual transfer. However, in particular cases, differences in the application range of concepts between English prepositions and their corresponding Chinese expressions may lead to positive conceptual transfer or negative conceptual transfer.

The analysis of the underlying causes of conceptual transfer has proved that the Chinese concepts play an important role in SLA. Under the guidance of Image Schema Theory, this study has also revealed that conceptual similarities and differences between Chinese and English arise from similar and different perspectives in the construal of reality or the physical world.

\section{Implications and limitations of this study}

There are three major implications of this corpus-based empirical study in terms of the theoretical development of conceptual transfer, the practice of English teaching and learning in the Chinese context, and research methodology. Firstly, this study provides implications for the theoretical development of conceptual transfer in the Chinese context. Secondly, this study has implications for the practice of English teaching and learning in the Chinese context in terms of the role of Chinese concepts. Thirdly, in terms of research methodology, since it is a corpus-based investigation of learners' authentic linguistic output, which is characterised by its authentic data and the replicable nature of this methodology.

Admittedly, this study has its limitations. Firstly, it only focused on the use of three English prepositions: "at", "in" and "on" according to the frequency of use and the evident conceptual transfer revealed by them. For the convenience of data analysis, peripheral situations or less-frequently used prepositions were left out. Secondly, in terms of levels of linguistic performance, this study just focused on the lexical level, leaving out conceptual transfer at the grammatical and the textual levels. Therefore, future studies need to take non-typical or peripheral prepositions into consideration to provide more convincing evidence for the existence of conceptual transfer not only at the lexical and grammatical levels, but also at the textual level. To wit, linguistic representations at different levels should be examined.

\section{References}

Aoyama, Katsura, Flege James E., Guion Susan G., Akahane-Yamada Reiko and Yamada Tsuneo. 2004. Perceived phonetic dissimilarity and L2 speech learning: The case of Japanese /r/ and English /1/ and /r/. Journal of Phonetics 32(2), 233-250. 
Casasanto, Daniel. 2009. When is a linguistic metaphor a conceptual metaphor? In: Evans, Vyvyan and Stephanie Pourcel (eds.), New Directions in Cognitive Linguistics, 127-145. Amsterdam/ Philadelphia: John Benjamins Publishing Company.

Gibbs, Raymond W. and Herbert L. Colston. 1995. The cognitive psychological reality of image schemas and their transformations. Cognitive Linguistics 6(4), 347-378.

Hancin-Bhatt, Barbara. 2000. Optimality in second language phonology: Codas in Thai ESL. Second Language Research 16(3), 201-232.

Ijaz, Helene I. 1986. Linguistic and cognitive determinants of lexical acquisition in a second language. Language Learning 36(4), 401-451.

Jarvis, Scott. 2011. Conceptual transfer: Crosslinguistic effects in categorization and construal. Bilingualism: Language and Cognition 14(1), 1-8.

Jarvis, Scott and Terence Odlin. 2000. Morphological type, spatial reference, and language transfer. Studies in Second Language Acquisition 22(4), 535-556.

Jarvis, Scott and Aneta Pavlenko. 2008. Crosslinguistic Influence in Language and Cognition. New York: Routledge.

Johnson, Mark. 1987. The Body in the Mind: The Bodily Basis of Meaning, Imagination, and Reason. Chicago: University of Chicago Press.

Koda, Keiko. 2000. Cross-linguistic variations in L2 morphological awareness. Applied Psycholinguistics 21(3), 297-320.

Kwon, Jihyun. 2003. Pragmatic transfer and proficiency in refusals of Korean EFL learners. Unpublished PhD dissertation, Boston University.

Lakoff, George and Mark Johnson. 1980. Metaphors We Live By. Chicago: University of Chicago Press.

Ma, Shuhong. 2010. Classification and acquisition of English spatial prepositions by EFL learners: The perspective of Categorization Theory. Journal of PLA University of Foreign Languages 33(4), 64-69.

Matthews, Stephen and Virginia Yip. 2003. Relative clauses in early bilingual development: Transfer and universals. In: Ramat, Anna G. (ed.), Typology and Second Language Acquisition, 39-81. Berlin/New York: Mouton de Gruyter.

Oakley, Todd. 2007. Image schemas. In: Geeraerts, Dirk and Hubert Cuyckens (eds.), The Oxford Handbook of Cognitive Linguistics, 214-235. Oxford: Oxford University Press.

Odlin, Terence. 1989. Language Transfer: Cross-linguistic Influence in Language Learning. Cambridge: Cambridge University Press.

Odlin, Terence. 2005. Crosslinguistic influence and conceptual transfer: What are the concepts? Annual Review of Applied Linguistics 25, 3-25.

Pavlenko, Aneta. 1999. New approaches to concepts in bilingual memory. Bilingualism: Language and Cognition 2(3), 209-230.

Pavlenko, Aneta. 2002. Bilingualism and emotions. Multilingua 21(1), 45-78. 
Pederson, Eric, Danziger Eve, Wilkins David, Levinson Stephen, Kita Sotaro and Gunter Senft. 1998. Semantic typology and spatial conceptualization. Language 74(3), 557-589.

Ringbom, Hakan. 2001. Lexical transfer in L3 production. In: Cenoz, Jasone, Hufeisen Britta and Urlike Jessner (eds.), Cross-linguistic Influence in Third Language Acquisition: Psycholinguistic Perspectives, 59-68. Clevedon: Multilingual Matters.

Gennari, Silvia P., Sloman Steven A., Malt Barbara C. and Tecumseh W. Fitch. 2002. Motion events in language and cognition. Cognition 83(1), 49-79.

Sinclair, John. 2004. Trust the Text: Language, Corpus and Discourse. London/New York: Routledge. von Stutterheim, Christiane. 2003. Linguistic structure and information organisation: The case of very advanced learners. EuroSLA Yearbook 3(1), 183-206.

Tamanaha, Masako. 2003. Interlanguage speech act realization of apologies and complaints: The performances of Japanese L2 speakers in comparison with Japanese L1 and English L1 speakers. Unpublished PhD dissertation, University of California, Los Angeles.

$\mathrm{Xu}$, Qingli, Liu Zhenqian and Cai Jinting. 2014. The influence of the categorization of reference objects on the use of the English spatial preposition "in" by Chinese learners. Foreign Language Teaching and Research 46(5), 723-734.

$\mathrm{Xu}$, Shen. 2001. Shuo Wen Jie Zi. Nanjing: Jiangsu Guji Publishing House.

Zhang, Huiping and Liu Yongbing. 2013. English preposition learning and conceptual transfer: Collocation and colligation of the most frequently used prepositions. Foreign Language Teaching and Research 45(4), 568-580.

Zhang, Huiping and Liu Yongbing. 2013b. The acquisition of English metaphorical prepositions and conceptual transfer from the perspective of linguistic relativity. Foreign Language Education 34(5), 51-55. 